showed that, in the worst predicted scenario (median LOS corresponding to 12 days), $1146 € /$ patient would be saved if ERAS protocols were applied.

Conclusion* Besides of achieving lower LOS and fewer readmission rates, implementation of an ERAS program in AOC surgery leads to valuable hospital savings. Therefore, ERAS should be the standard practice for AOC surgeries.

\section{ROLE OF INTEGRINS IN THE METASTATIC SPREAD OF HIGH-GRADE SEROUS OVARIAN CANCER}

${ }^{1} S$ Krajnak*, ${ }^{2} \mathrm{~J}$ Jäkel, ${ }^{1} \mathrm{~K}$ Anic, ${ }^{1}$ AS Heimes, ${ }^{1} \mathrm{~V}$ Linz, ${ }^{1} \mathrm{R}$ Schwab, ${ }^{1} \mathrm{E}$ Vacca, ${ }^{1} \mathrm{M}$ Schmidt, ${ }^{1} \mathrm{~A}$ Hasenburg, ${ }^{2} \mathrm{~W}$ Roth, ${ }^{1} \mathrm{~W}$ Brenner, ${ }^{1} \mathrm{M}$ Battista. 'University Medical Centre of the Johannes Gutenberg University Mainz, Department of Gynaecology and Obstetrics, Mainz, Germany; ${ }^{2}$ University Medical Centre of the Johannes Gutenberg University Mainz, Department of Pathology, Mainz, Germany

\subsection{6/ijgc-2021-ESG0.384}

Introduction/Background* In high-grade serous ovarian cancer (HGSOC) an early intraperitoneal metastatic spread is common which determines the therapeutical approach and prognosis. Integrins may be involved in metastatic spread of HGSOC. In this study, integrin expression was examined in primary tumour and metastases of HGSOC.

Methodology The expression of integrin $\alpha 2, \alpha 4, \alpha 5, \alpha 6$, and $\beta 1$ was assessed by immunostaining in tumour samples of the ovary, omentum, and peritoneum of each patient. Differences in integrin expression among tumour localisations and their association with clinicopathological parameters were examined by Fisher's exact test. The impact of integrin expression on progression-free survival (PFS) and overall survival (OS) was examined by Cox regression and Kaplan-Meier analyses.

Result(s)* 113 tumour samples of 40 HGSOC patients were examined. The expression of the integrins did not differ between the three tumour localisations (all p-values >0.05) with the exception of the expression of integrin $\beta 1$ in primary tumour and omentum $(77.5 \%$ versus $57.5 \%, p=0.014)$. Significant differences were also observed with respect to high expression of integrin $\alpha 4$ in primary tumour and omentum $(52.5 \%$ versus $47.5 \%, \mathrm{p}=0.008)$ and primary tumour and peritoneum $(52.5 \%$ versus $47.5 \%, \mathrm{p}=0.050)$. High expression of integrin $\alpha 4$ in peritoneum was associated with poorer PFS $(\mathrm{HR}=2.02 \quad 95 \%$ CI 1.01-4.05, $\mathrm{p}=0.047)$, younger age $(p=0.047)$ and death $(p=0.046)$. Median PFS in patients with

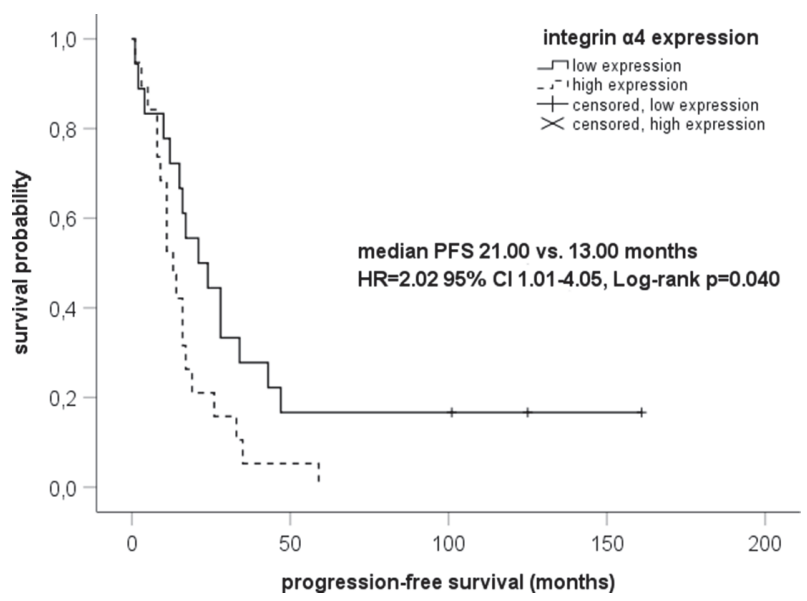

Abstract 317 Figure 1

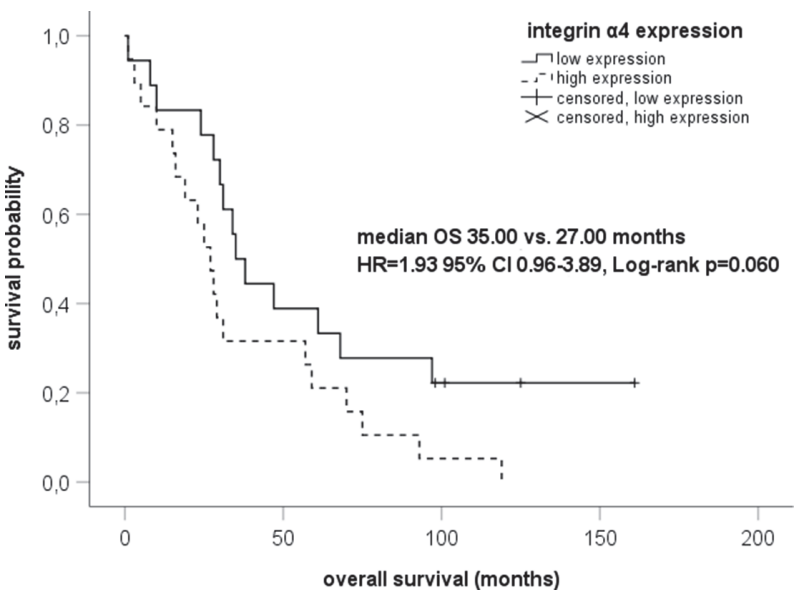

Abstract 317 Figure 2

high expression of integrin $\alpha 4$ was 13.00 months whereas median PFS in patients without high expression of integrin $\alpha 4$ was 21.00 months $(p=0.040)$. Expression of integrin $\alpha 2, \alpha 5$, $\alpha 6$, and $\beta 1$ did not correlate with PFS or OS.

Conclusion* Expression of integrin $\alpha 4$ may be altered during the metastasic spread of HGSOC and affect the prognosis. Expression of integrin $\alpha 2, \alpha 5, \alpha 6$, and $\beta 1$ did not reveal any prognostic value in HGSOC, even if expression of integrin $\beta 1$ differed between primary tumour and omental metastases.

\section{ADVANCED OVARIAN CANCER SURVIVAL RATES IN A MEDITERRANEAN POPULATION: AN 8-YEAR REAL- WORLD NATIONAL ANALYSIS OF THE MALTESE ISLANDS}

V Cassar*, MC Tabone, MP Agius, Y Muscat Baron. Mater Dei Hospital, Obstetrics and Gynaecology Department, MSD2090, Malta

\subsection{6/ijgc-2021-ESGO.385}

Introduction/Background* Surgical management of advanced ovarian cancer(AOC) is considered technically challenging. In order to achieve the required outcomes a centre requires expertise and flow of cases. ESGO recommends that accredited centres perform at least 24 complete cytoreductive(CCR) surgeries per year, with a minimum of 12 complete primary debulking surgeries(PDS).

Malta is a small European island with a population of 520,000 , seeing an average of 40 ovarian cancer cases per year, 20 of which are AOC. Being a small island, Malta faces a different reality and numerous challenges compared to other European countries.

Methodology This is a retrospective study, where all abnormal histology results suggestive of abnormal ovarian pathology during the time period of 2008-2016, processed by the Pathology Department in Mater Dei Hospital Malta were assessed. Furthermore, data was collected by reviewing the electronic medical records, histology results and imaging.

Result(s)* Over the 8 year period,146 new patients were diagnosed with advanced ovarian cancer. The median age at diagnosis was 64.5(33-91)years. 105/146(71.9\%) women presented with FIGO stage III and 41/146(28.1\%) with stage IV disease. Overall survival(OS) 32.87 months with a 1 -year survival of $70.55 \%$ and 5 -year survival of $20.28 \%$. Treatment was offered to $128 / 146(87.67 \%), \quad 103(80.47 \%)$ patients were offered 